\title{
Loop Ileostomy Closure: Comparison of Cost Effectiveness between Suture and Stapler
}

\author{
Karoline Horisberger • Guido Beldi • \\ Daniel Candinas
}

Published online: 15 September 2010

(C) Société Internationale de Chirurgie 2010

\begin{abstract}
Background Closure of loop ileostomy can be safely performed using sutures or staplers. The aim of the present study was to compare the cost effectiveness of three different techniques.

Methods A total of 128 consecutive patients who underwent closure of loop ileostomy between January 2002 and December 2008 were analyzed retrospectively. The primary outcome parameter was operative cost.

Results Closure of ileostomy was performed in 66 patients with hand-sewn anastomosis, in 25 patients with stapler only, and in 37 patients with a combination of stapler and suture. There were no differences in terms of early and late postoperative complications. Operative time was significantly longer for "suture only" (101.4 $\pm 26 \mathrm{~min})$ than for "stapler/ suture" (-4.9 $\mathrm{min})$ and "stapler only" $(-17.8 \mathrm{~min})$; the difference between the three groups is significant $(p=$ 0.05). Duration of hospital stay was not different among the three groups. Operative costs with "stapler/suture" $(1,755.9 \pm 355.6$ EUR) were significantly higher than with "suture only" $(-254$ EUR; $p=0.001)$ and "stapler only" ( -236 EUR; $p=0.005)$.

Conclusions Operative time using the stapler only is significantly shorter than with hand-sewn anastomosis or combinations of stapler and suture. Operative costs are significantly higher for a procedure that includes suture and stapler.
\end{abstract}

K. Horisberger · G. Beldi $(\bowtie) \cdot$ D. Candinas

Department of Visceral Surgery and Medicine, Bern University

Hospital, University of Bern, 3010 Bern, Switzerland

e-mail: guido.beldi@insel.ch

\section{Introduction}

The closure of a defunctioning loop ileostomy is traditionally undertaken 6-12 weeks after initial surgery $[1,2]$. Routine closure of the stoma can be performed using either a hand-sewn end-to-end anastomosis or various techniques using staples [3-5]. The theoretical advance of stapled anastomosis over the other techniques is the potentially shorter operative time and the larger luminal diameter, thereby being less associated with postoperative small bowel obstruction [3]. However, in terms of patient safety, no significant differences have been shown between stapled and sutured anastomosis $[4,6]$. Given the similar results with different techniques, we analyzed the cost-effectiveness of three different procedures. With the present study, we compared operative and total costs between hand-sewn and two variants of stapled anastomosis. Secondary outcome parameters include overall morbidity, operating time, and duration of hospital stay.

\section{Patients and methods}

All consecutive patients undergoing closure of loop ileostomy between January 2002 and December 2008 were included in this retrospective study. Loop ileostomy during the primary operation was created using the terminal part of the ileum at a premarked site in the right iliac fossa. Before closure of the ileostomy, all patients underwent a contrast study (an antegrade water-soluble enema) of the colon to exclude strictures of the anastomosis. Surgical fellows performed all surgical procedures of closure. 
Surgical techniques

At the beginning of the operation, the site was disinfected and draped. After skin incision, the ileostomy was inverted and the enterotomy was closed temporarily with a Prolene suture. The operative site was disinfected again and redraped to reduce the incidence of surgical site infections. Three groups of ileostomy closure were compared: group 1: suture only, group 2: stapling only (double stapling), and group 3: combination of stapling and suture. The surgical procedure of ileostomy closure was chosen according to the surgeon's preference.

\section{Group 1: suture only}

After resection of the temporarily closed ileostomy, the anastomosis was performed using a Polydioxanone 4-0 single-layer continuous suture (Fig. 1).

\section{Group 2: stapled only}

The antimesenteric borders of the ileum were approximated with a stay suture after mobilizing the spout to perform side-to-side (functional end-to-end) anastomosis. Anastomosis was performed using two GIA 55- or 75-mm staplers, one for the anastomosis and a second stapler cartridge for cross-stapling the apex of the loop (Ethicon, Neuchâtel, Switzerland) (Fig. 2).

\section{Group 3: stapler/suture}

The stoma limbs were first resected with two stapling devices, GIA $55 \mathrm{~mm}$. A stapled side-to-side anastomosis was then performed with one device and closure of the enterotomy by hand-suture using Polydioxanone 4-0 suture (Fig. 3).

Closure of the fascia was performed by continuous Polydioxanone 1 suture and the skin was closed with interrupted Prolene 4-0 sutures or clips.
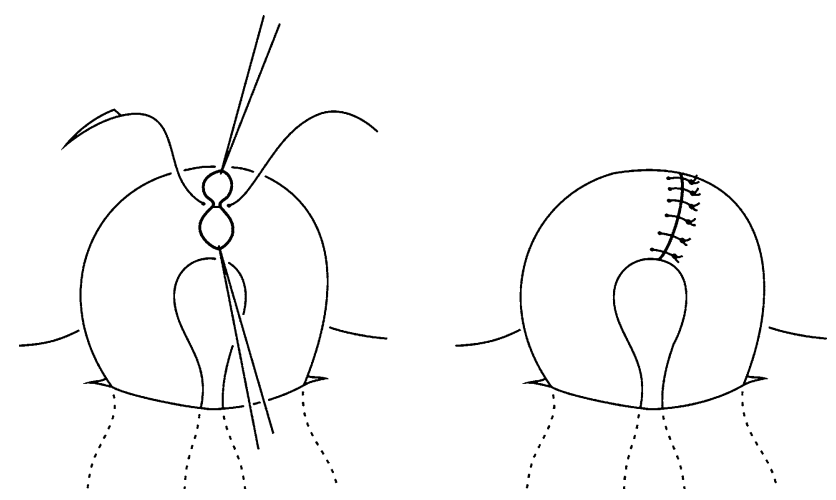

Fig. 1 Suture-only technique

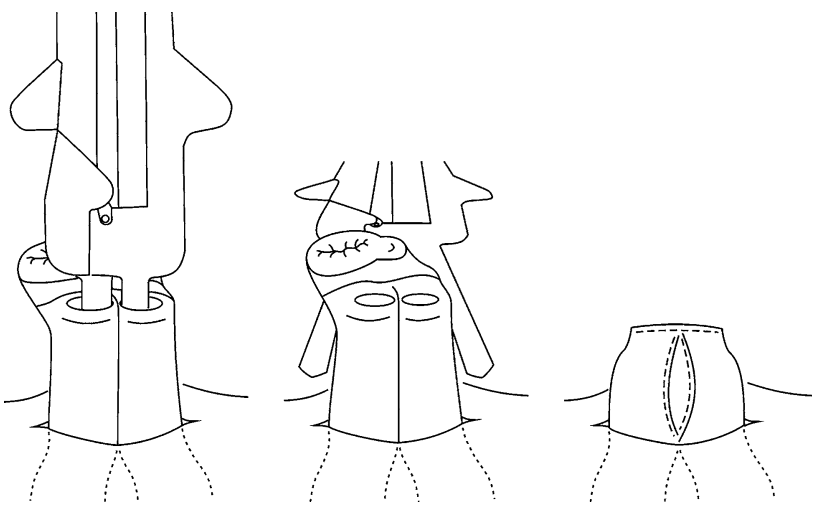

Fig. 2 Stapler only technique
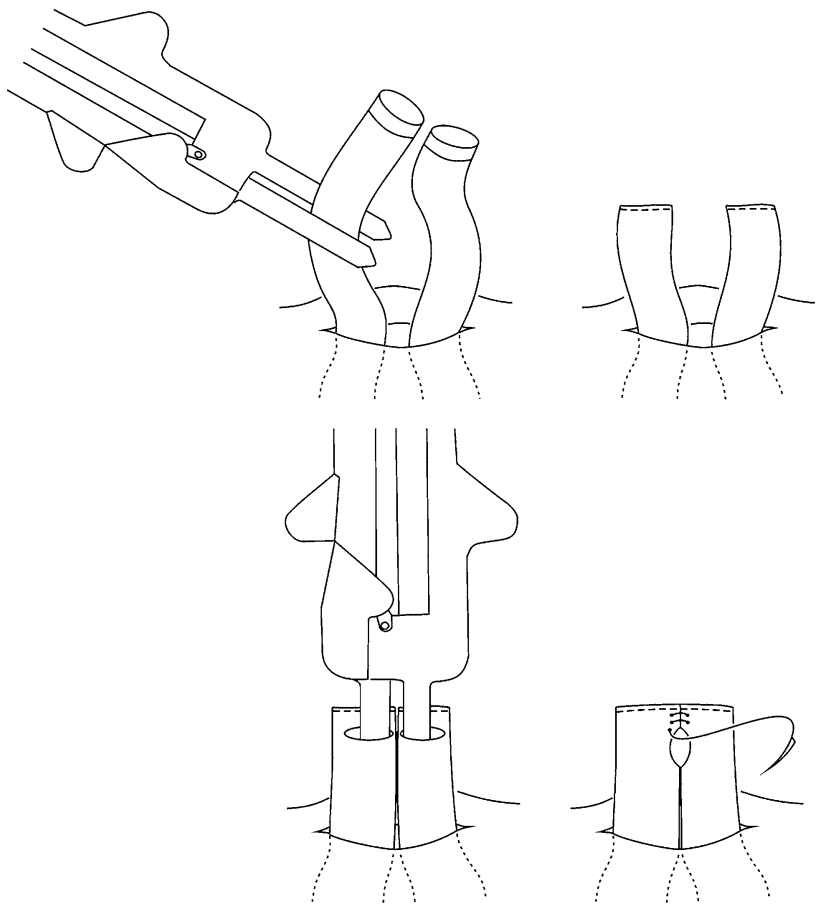

Fig. 3 Stapler/suture technique

Analysis

Overall operative time (skin-to-skin) was assessed. The time for anesthesia was not included in the analysis. Recovery of intestinal function was defined as the time taken from the operative day until the first bowel movement and until the intake of solid foods. Postoperative subileus/ileus was defined as delay of feeding progression up to postoperative day 5 or the requirement of a gastric tube. Anastomotic leakage was defined as a radiologic finding of fistula or fluid collection with clinical symptoms leading to reoperation. Diarrhea was defined as loss of liquid stool requiring additional liquid substitution. Surgical site infection was defined according to the Centers for Disease Control and Prevention criteria [7]. 


\section{Cost analysis}

The length of the operation was converted to costs (EUR) calculated with 14.57 EUR per min of operative time. Material costs were calculated as costs for the anastomosis (suture and stapler devices). Total costs of the operation were defined by the sum of costs of operative time and material costs for the anastomosis. The cost of one day of hospitalization was 463.7 EUR. Total costs were the sum of the operative costs and the costs for the hospital stay.

\section{Statistical analysis}

Statistical analysis was performed with SPSS software (version 15.0; SPSS, Chicago, IL). Significance was set at $p<0.05$. For nonparametric values, Kruskal-Wallis tests were performed among the three groups. For analysis between two groups, Mann-Whitney $U$-tests were performed. For parametric values, Pearson chi-square tests were performed.

\section{Results}

A total of 128 consecutive patients ( 82 male, 46 female) were included in the study. Patient characteristics are shown in Table 1. Intraoperative results and postoperative complications are shown in Table 2. Mean operative time was significantly different among the three groups.

There was no difference in time elapsed until the first stool and until intake of solid foods among the three groups. A total of $21.9 \%$ of the patients $(n=25)$ had a postoperative subileus/ileus; $10.9 \%(n=14)$ had diarrhea. Postoperative bowel obstruction occurred in $12 \%$ of the patients after stapled anastomosis compared to $22.7 \%$ after sutured closure. One patient with bowel obstruction after hand-sewn anastomosis needed a reoperation. Postoperative rectal bleeding occurred in four patients, one requiring transfusion of red blood cells.

Average operative time with "stapler only" was $17.8 \mathrm{~min}$ shorter than "suture only" and 12.9 min shorter than "suture/stapler" (Table 2). The costs associated with operative time were significantly higher for "suture only" compared with "stapler only" $(p=0.03$; Table 3$)$. The material costs for the anastomosis were significantly higher for "stapler only" than for "suture only" $(p<0.001)$. The total costs of the surgical procedure (including costs for operative time and technical devices) for "stapler/suture" were significantly higher than for the other groups (Fig. 4).

The length of postoperative hospital stay was not different among the groups (Table 3). Overall costs (including operation costs and hospital stay) were significantly lower for "stapler only" than for "stapler/suture" (Fig. 5). The difference of "stapler only" to "suture only" did not reach the level of significance.

\section{Discussion}

Operative costs for closure of loop ileostomy were not different between "stapler only" and "suture only." Suturing reduces material costs, whereas stapling reduces costs associated with operative time. The combination of suture and stapler is associated with higher material costs and no significant reduction in operative time, and it is therefore more expensive.

The incidence of postoperative complications was not different between the three groups. A higher rate of small bowel obstruction was observed after sutured anastomosis; however, it did not reach statistical significance. The lower level of postoperative bowel obstruction after stapled anastomosis seems to confirm the suggestion that the edema at a narrowed hand-sewn anastomosis creates a smaller lumen diameter $[3,8]$. Intestinal hemorrhage is a
Table 1 Patient characteristics and initial operations

\begin{tabular}{lllll}
\hline Characteristic & $\begin{array}{l}\text { Sutured } \\
(n=66)\end{array}$ & $\begin{array}{l}\text { Stapler } \\
(n=25)\end{array}$ & $\begin{array}{l}\text { Stapler/suture } \\
(n=37)\end{array}$ & $p$ Value \\
\hline Gender, male/female & $44 / 22$ & $13 / 12$ & $26 / 11$ & 0.304 \\
Age, years (mean \pm SD) & $57.2 \pm 15.2$ & $57.0 \pm 15.0$ & $58.5 \pm 16.1$ & 0.903 \\
BMI, kg/m & $24.6 \pm 4.9$ & $23.3 \pm 3.2$ & $25.0 \pm 4.6$ & 0.589 \\
(low) Anterior resection & 30 & 17 & 16 & 0.121 \\
Proctocolectomy & 4 & 2 & 6 & 0.177 \\
Hemicolectomy & 20 & 4 & 8 & 0.277 \\
Rectovaginal fistula & 3 & 1 & 1 & 0.915 \\
Crohn's disease & 4 & 0 & 3 & 0.323 \\
Stump abdominal trauma & 1 & 0 & 0 & 0.623 \\
Paralytic ileus (pancreatitis, & 4 & 2 & 2 & 0.959 \\
$\quad$ intra-abdominal bleeding, & & & & \\
and so on) & & & & \\
\hline
\end{tabular}


Table 2 Results

Sutured $(n=66)$

Stapler $(n=25)$

Stapler/suture

$p$ Value

$(n=37)$

\section{Results}

Operative time, min (mean \pm SD)

$\begin{array}{ll}101.4 \pm 26 & 83.6 \pm 15.0 \\ 8.2 \pm 3.7 & 7.0 \pm 2.7 \\ 3.08 \pm 1.3 & 2.9 \pm 1.2 \\ 4.85 \pm 1.98 & 4.6 \pm 2.1 \\ & \\ 15(22.7 \%) & 3(12 \%) \\ 6(9.1 \%) & 4(16 \%) \\ 0 & 2(8 \%) \\ 1(1.5 \%) & 1(4 \%) \\ 0 & 0 \\ 1 & 0\end{array}$

$96.5 \pm 24.3$

0.005

Hospital stay, days (mean $\pm \mathrm{SD}$ )

First bowel movement, days (mean \pm SD)

First solids, days (mean \pm SD)

Complications

Bowel obstruction

Diarrhea

Postoperative bleeding

Surgical site infection

Anastomotic leakage

0

$8.0 \pm 3.0$

0.250

$2.9 \pm 1.2$

0.657

$4.9 \pm 1.6$

0.513

Reoperation

Table 3 Costs for anastomoses

\begin{tabular}{lccc}
\hline Costs & $\begin{array}{l}\text { Suture only } \\
(n=66)\end{array}$ & $\begin{array}{l}\text { Stapler only } \\
(n=25)\end{array}$ & $\begin{array}{c}\text { Stapler/suture } \\
(n=37)\end{array}$ \\
\hline Operative time, min (mean \pm SD) & $101.4 \pm 26.0$ & $83.6 \pm 15.0$ & $96.5 \pm 24.3$ \\
Costs for operative time, EUR (mean \pm SD) & $1,476.1 \pm 378.4$ & $1,217.2 \pm 217.9$ & $1,404.8 \pm 353.7$ \\
Costs for material, EUR (mean \pm SD) & $25.5 \pm 57.8$ & $301.9 \pm 46.7$ & $346.7 \pm 96.3$ \\
Total costs for operation, EUR (mean \pm SD) & $1,501.9 \pm 384.8$ & $1,519.3 \pm 202.9$ & $1,755.9 \pm 355.6$ \\
Hospital stay, days (mean \pm SD) & $8.2 \pm 3.7$ & $7.0 \pm 2.7$ & 0.005 \\
Overall cost, EUR (mean \pm SD) & $5,355.7 \pm 1,867.1$ & $4,703 \pm 1,161.1$ & $5,454.4 \pm 1,511.9$ \\
\hline
\end{tabular}

${ }^{a}$ Kruskal-Wallis test

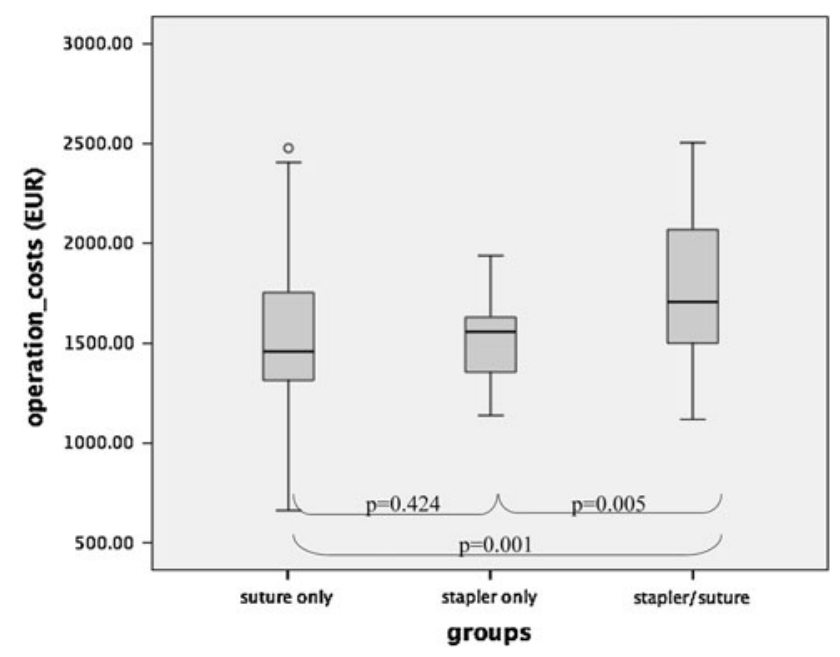

Fig. 4 Comparison of total operative costs, euros (EUR); (MannWhitney $U$-tests)

potentially significant complication of stapled ileostomy closure and was observed in four of our patients [9-11].

The present data reveal that the combination of stapler and suture is the most expensive closure technique and is

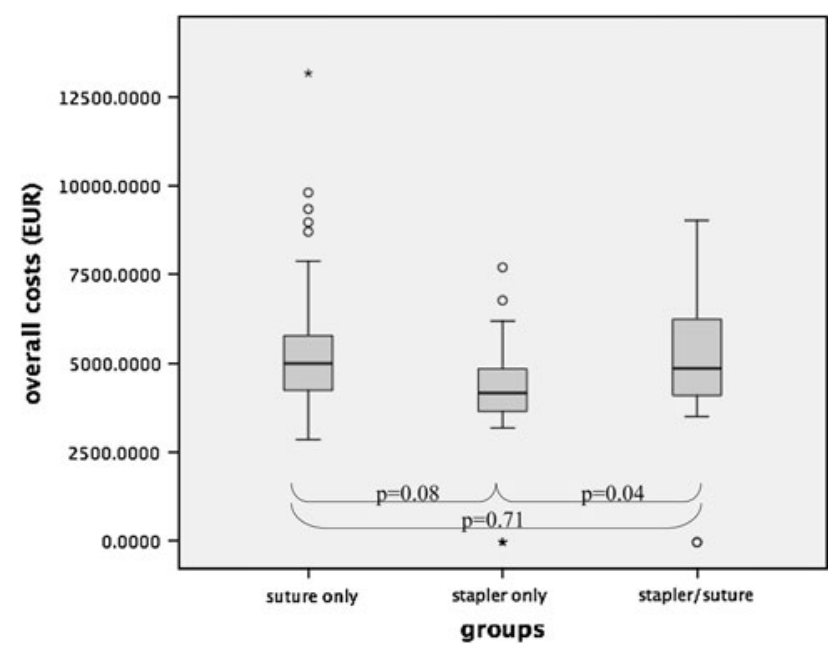

Fig. 5 Comparison of total overall costs (EUR); (Mann-Whitney $U$-tests)

not associated with reduction of operative time. Further this technique is not associated with a reduction of complications and it seems unlikely that such an approach would reveal advantages in terms of patient safety and total cost 
in a prospective trial. However, the present study was underpowered in order to find significant differences in terms of relevant postoperative complications. Previous studies that addressed these clinical issues have not shown significant differences in clinical outcome between various techniques $[3,12]$, whereas a recently published singlecenter study shows a reduction of postoperative morbidity after stapled ileostomy closure [5]. A further restriction of the study might be that in the retrospective setting the specific procedures were mostly carried out by the same surgeons, as they preferred one technique. However, as over the years many different surgeons took part in the analysis we assume that the sheer number balanced those differences that actually could cause a bias. However, the groups are underpowered to correctly address this question.

The difference in the time required for stapling and suturing determines if stapling can be cost-effective. Previous studies have shown that a reduction of operating time by $15 \mathrm{~min}$ with stapled anastomoses reduced overall cost per case [13]. However, a reduction by 4 min was not associated with a reduction of cost [3]. In our institution, the saved time of $17.8 \mathrm{~min}$ did not decrease overall cost. As costs per minute of operation and costs of material differ between countries and hospitals, the cost-effectiveness of stapling has to be evaluated by each specific institution.

In conclusion, combinations of stapling and suture do not reduce operative time or material cost. The costeffectiveness of ileostomy closure by stapling only versus suture only depends on the amount of operative time saved and the material costs, and has to be determined by the individual institution.

Conflict of interest None.

\section{References}

1. Tjandra JJ, Fazio VW, Milsom JW et al (1993) Omission of temporary diversion in restorative proctocolectomy-is it safe? Dis Colon Rectum 36:1007-1014

2. Chand M, Nash GF, Talbot RW (2008) Timely closure of loop ileostomy following anterior resection for rectal cancer. Eur J Cancer Care 17:611-615

3. Hasegawa H, Radley S, Morton DG et al (2000) Stapled versus sutured closure of loop ileostomy: a randomized controlled trial. Ann Surg 231:202-204

4. Mansfield SD, Jensen C, Phair AS et al (2008) Complications of loop ileostomy closure: a retrospective cohort analysis of 123 patients. World J Surg 32:2101-2106

5. Shelygin YA, Chernyshov SV, Rybakov EG (2010) Stapled ileostomy closure results in reduction of postoperative morbidity. Tech Coloproctol 14:19-23

6. Allal AS, Kahne T, Reverdin AK et al (2004) Radioresistancerelated proteins in rectal cancer. Proteomics 4:2261-2269

7. Beldi G, Bisch-Knaden S, Banz V et al (2009) Impact of intraoperative behavior on surgical site infections. Am J Surg 198:157-162

8. Feinberg SM, McLeod RS, Cohen Z (1987) Complications of loop ileostomy. Am J Surg 153:102-107

9. Williams LA, Sagar PM, Finan PJ et al (2008) The outcome of loop ileostomy closure: a prospective study. Colorectal Dis $10: 460-464$

10. Fischer MG (1976) Bleeding from stapler anastomosis. Am J Surg 131:745-747

11. Tuchmann A, Dinstl K, Strasser K et al (1985) Stapling devices in gastrointestinal surgery. Int Surg 70:23-27

12. Leung TTW, Mac Lean AR, Buie WD et al (2008) Comparison of stapled versus handsewn loop ileostomy closure: a metaanalysis. J Gastroinetst Surg 12:939-944

13. Hull TL, Kobe I, Fazio VW (1996) Comparison of handsewn with stapled loop ileostomy closures. Dis Colon Rectum 39:1086-1089 